\title{
Zone mapping application for precision-farming: a decision support tool for variable rate application
}

\author{
Xiaodong Zhang $\cdot$ Lijian Shi $\cdot$ Xinhua Jia $\cdot$ George Seielstad \\ Craig Helgason
}

Published online: 12 August 2009

(C) The Author(s) 2009. This article is published with open access at Springerlink.com

\begin{abstract}
A web-based decision support tool, zone mapping application for precision farming (ZoneMAP, http://zonemap.umac.org), has been developed to automatically determine the optimal number of management zones and delineate them using satellite imagery and field data provided by users. Application rates, such as of fertilizer, can be prescribed for each zone and downloaded in a variety of formats to ensure compatibility with GPS-enabled farming equipment. ZoneMAP is linked to Digital Northern Great Plains, a web-based application which hosts an archive of satellite imagery, as well as high resolution imagery from airborne sensors. Management zones created by ZoneMAP mapped natural variation of the soil organic matter and other nutrients relatively well and were consistent with zone maps created by traditional means. The results demonstrated that ZoneMAP can serve as an effective and easy-to-use tool for those who practice precision agriculture.
\end{abstract}

Keywords Fuzzy c-means - Management zone $\cdot$ Zone map $\cdot$ Remote sensing · Variable rate application

\section{Introduction}

Given increasing prices of chemicals and fuel, and increasing awareness of the need to preserve our natural environment, producers rely more and more on precision farming to

X. Zhang $(\bowtie) \cdot$ G. Seielstad $\cdot$ C. Helgason

Northern Great Plains Center for People and the Environment, University of North Dakota,

Stop 9011, Grand Forks, ND 58202-9011, USA

e-mail: zhang@aero.und.edu

L. Shi

National Satellite Ocean Application Service, 8 Dahuisi Road, 100081 Beijing, China

X. Jia

Agriculture and Biosystems Engineering, North Dakota State University, PO Box 6050, Fargo, ND 58108-6050, USA 
reduce economic and environmental costs. Precision farming is concerned inter alia with the ability to vary rates of application and precisely apply inputs based on actual crop needs. Developing a management zone map is essential for effective variable rate applications. To develop a zone map, normally three factors need to be considered: information to be used as a basis for creating zones, procedure to be used to process the information (i.e., classification), and the optimal number of zones that a field should be divided into (Fridgen et al. 2004). Efficient and easy-to-use tools that address all these factors are needed to provide a technology delivery mechanism, the lack of which has been identified as the major obstacle to the wide adoption of precision farming (Zhang et al. 2002).

A multitude of spatial information or a combination of them can be used to derive the variability in a field. The physical and chemical properties of the soil determined from manual soil sampling are often used in fertilizer recommendations for crops. Normally a large number of samples, and hence large expense in cost and time, is needed to achieve statistical significance among samples in determining management zones (Franzen et al. 2002). Spatial variability in yields has been considered as another useful indicator in determining variable rate nutrient management (Johnson et al. 2003). The yield variation not only reflects within-field variation in soil productivity potential (Brock et al. 2005), but also provides an indication of the nutrient level for the following spring if crop residues are left to decay. The experiences of farmers are also important. Fleming et al. (2000) evaluated farmer-developed management zone maps and concluded that soil color from aerial photographs, topography, as well as the farmer's past management experience are effective in developing variable rate application maps.

Spatial imagery in agriculture has been used for crop management since 1929 when aerial photography was used to map soil resources (Seelan et al. 2003). Remote sensing for precision agriculture is based on the relationships of surface spectral reflectance with various soil properties and crop characteristics (Moran et al. 1997). Spectral reflectance of the soil or crops that were measured in the laboratory (Daniel et al. 2004), from hand-held devices (Read et al. 2002), from aerial photography (Fleming et al. 2000), and from satellite observations (Bhatti et al. 1991; Salisbury and D'Aria 1992; Seelan et al. 2003; Sullivan et al. 2005) have been widely used in developing variable rate application maps. Multi-temporal images by satellites within a growing season have also been used to study within-field variability (Bégué et al. 2008). Despite these theoretical advances and successful applications, access to and use of remote sensing data by end users require considerable technical knowledge about computing and remote sensing, and remain as a challenge (Moreenthaler et al. 2003; Zhang et al. 2002).

Unsupervised classification algorithms are often used to divide a field into zones because it is normally assumed that the users may have no a priori knowledge of what information or areas should be used for training. Fuzzy-c-means, an unsupervised classification algorithm, has been shown to be effective in delineating a field into management zones for a variety of applications (Burrough 1989; Burrough et al. 1992; Lark and Stafford 1997; Odeh et al. 1992).

Determining the most appropriate number of zones is difficult in the interpretation of unsupervised classification. Odeh et al. (1992) suggested the use of fuzziness performance index (FPI) and normalized classification entropy (NCE) to determine the optimal number. Theoretically, the best classification occurs when membership sharing (FPI) and the amount of class disorganization (NCE) is at a minimum with the least number of classes used. However, sometimes NCE and FPI do not converge and the optimal number of zones suggested by one parameter is significantly different from the one suggested by the other (Brock et al. 2005). 
Significant advancement of knowledge has been gained in acquisition of spatial data and their analysis and interpretation. However, Zhang et al. (2002) reported in 2002 that the adoption rate for precision agriculture was slower than expected. While the cost, lack of perceived benefit and conservatism among farmers have contributed to the slowness, the problem in delivering the necessary technologies to farmers was identified as the major obstacle (Zhang et al. 2002). Although many GIS packages contain the functions necessary for creating potential management zones from spatial data, they can be cumbersome to use and require considerable time to learn.

Fridgen et al. (2004) developed a management zone analysis (MZA) tool for sub-field management zone delineation. This Windows-based software is easy to use and effective in delineating management zones. However, it places a stringent requirement on data preparation, and all the input layers, vector or raster, have to be gridded into common grid cells. In addition, MZA and many other application tools fail to address a major issue that has hampered the wide adoption of precision agriculture: access to data, especially those from remote sensing platforms.

A web-based decision support tool, Zone Mapping Application for Precision Farming (ZoneMAP), has been developed to provide users with a tool that is simple to use while at the same time addresses the three factors mentioned above. ZoneMAP uses the fuzzyc-means (FCM) algorithm for classifying fields into zones. It has seamless access to an archive of remote sensing data spanning the past 30 years with automatic procedures streamlining format conversion, re-projection, and gridding of data from various sources. It also has a capability to automatically determine the optimal number of zones based on the available data.

In this paper, the development of the algorithms used in ZoneMAP for classification and automatic determination of optimal number of zones is reported. The image database is described and examples of applying ZoneMAP to two production farm fields are evaluated.

\section{Methods}

\section{Classification algorithm for ZoneMAP}

Fuzzy c-means was chosen as the clustering algorithm for ZoneMAP. It is basically the same approach as the one used by Fridgen et al. (2004) except for the method of estimating the measure of similarity between observations and centers of the zones that are to be determined. Typically, the measure of similarity can be estimated using Euclidean distance, diagonal distance or Mahalanobis distance. Since the Euclidean distance algorithm requires variables to be of equal variances and non-correlated (rarely true in reality) only the latter two algorithms were implemented.

The diagonal distance algorithm compensates for the difference in variances of different variables, but it is still sensitive to correlated variables. The Mahalanobis distance algorithm accounts for situations where input variables are statistically dependent with unequal variance. Since it relies on a variance-covariance matrix for weighting, which has to be calculated for all the input variables, it is more computationally intensive than estimating diagonal distance. Both the diagonal and Mahalanobis distance algorithms gave very similar results during the testing. Occasionally, the final classification with Mahalanobis distance may vary depending on the initial values assigned to the cluster centers, a problem also reported by Fridgen et al. (2004). The diagonal distance algorithm is faster and therefore suitable for a web-based application, such as ZoneMAP. By default, the diagonal 
distance algorithm is used in classification. For estimating diagonal distance, instead of adjusting the estimate by the variance of the related variables, the input variables are reshaped before computation such that they all have a mean of zero and a unit variance. This process does not affect the classification results, but it enhances the speed of performance.

Algorithm for optimal number of zones

The method used to evaluate classification success was to estimate how much withinzone variability is reduced for a number $(n)$ of zones as compared with $n-1$ zones. Generally, the percentage of total within-zone variability with respect to the total initial variability decreases as the number of zones increases as shown in Fig. 1. A similar trend for the variance reduction was found by Brock et al. (2005). The total within-zone variance decreases rapidly initially and then approaches an asymptotic value slowly as the number of zones continues to increase. The optimal number of zones is therefore decided as the number that reduces the variance significantly as compared to the initial variability, yet changes little when the number of zones is further increased. Two criteria were determined through trial and error, which seem to be able to capture this turning point in a relatively consistent manner: (1) overall reduction of variance is $>50 \%$; and (2) consecutive reduction of variance is $<20 \%$ or the trend is broken, i.e., within-zone variability increases instead of decreases. For the case shown in Fig. 1, the optimal number of zones would be 5 .

\section{Design of ZoneMAP}

ZoneMAP was designed for end users like farmers, ranchers or extension specialists to implement precision agriculture; therefore the ease of use is important. Also important is the access to data, especially remote sensing observations which have been shown to be extremely effective in capturing field variability (e.g., Seelan et al. 2003; Sullivan et al. 2005).

Fig. 1 The total within-zone variability as a percentage of initial variance normally decreases with the number of zones

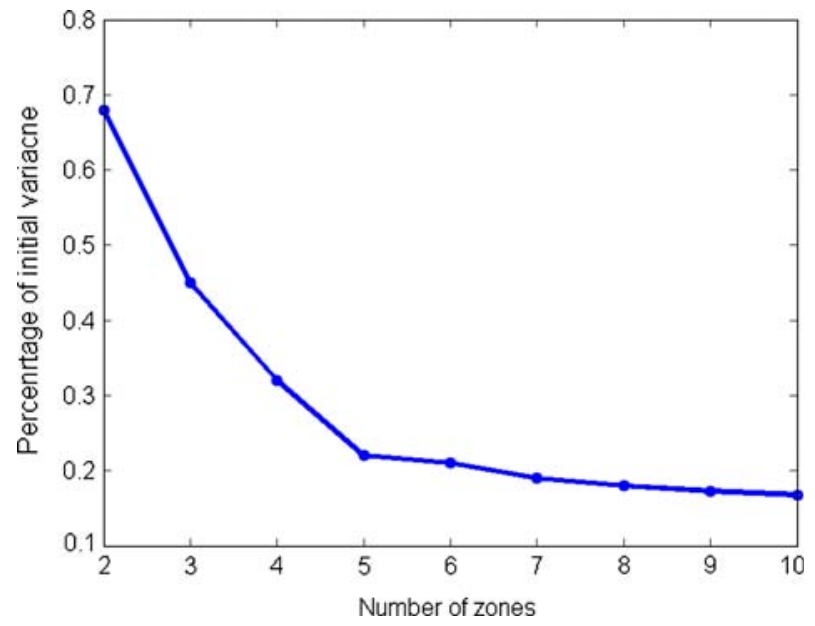




\section{Remote sensing imagery}

An archive of remote sensing imagery spanning over 30 years over the northern Great Plains of the USA, including North and South Dakota, Minnesota, Montana, Wyoming and Idaho was collected. Data include medium resolution $(20-250 \mathrm{~m})$ multi-spectral images from satellite sensors of Landsat multi-spectral scanner (MSS), thematic mapper (TM) and enhanced thematic mapper plus (ETM+), advanced space borne thermal emission and reflection radiometer (ASTER), moderate resolution imaging spectro-radiometer (MODIS), surface relief from shuttle radar topography mission (SRTM) and high resolution (1-2 m) images from AeroCam, a multi-spectral airborne camera that was developed and operated by the upper midwest aerospace consortium (UMAC, http://www.umac.org). These data are publicly available through Digital Northern Great Plains (http://dngp.umac.org), to which ZoneMAP is internally linked for seamless access to this digital resource.

To ensure consistency in temporal and spatial comparison, all satellite images have been atmospherically corrected to remove unwanted signals due to scattering and absorption by the atmosphere. The final product after the atmospheric correction is the reflectance on the ground. The AeroCam sensor has been calibrated at the NASA Ames Research Center to determine its spectral and radiometric characteristics. However, at a typical altitude of 2-3 km, the atmospheric contribution to the AeroCam signal is significantly reduced. Therefore, no atmospheric correction is performed for AeroCam images and only radiometric correction is applied. The final product for AeroCam is the radiance at the height of the aircraft instead of reflectance at ground level.

A canopy's reflectance will change during a growing season as vegetation goes through stages of first growth, maturity, reproduction and senescence. Vegetation indices, such as normalized differential vegetation index (NDVI) estimated using reflectance measurements at the red and near-infrared (NIR) wavelengths, or Green NDVI estimated by replacing the red with the green, have been developed to track the vigor of plants and have been used widely for developing management zones (Metternicht 2003; Moran et al. 1997). While NDVI has been used as an indicator of biomass, GNDVI was suggested as a better estimate of Chlorophyll concentration (Gitelson et al. 1996). GNDVI has been used for high biomass crops because of saturation of Chlorophyll in the red wavelength (Gitelson et al. 1996). ZoneMAP will estimate NDVI and GNDVI on-the-fly if a user chooses the vegetation index as a basis for classification.

\section{Image processing}

For management zones to be representative, various factors affecting the soil characteristics and potential productivity need to be considered. This often entails the use of data from different sources, of different ground sampling distances, and with different formats and projections. Before being combined for further analysis, different data need to be projected onto a common grid, which often involves sub-setting, re-projecting and re-sampling procedures.

Typically, a remote sensing image covers a much bigger area than a farm field. Instead of processing the entire image, ZoneMAP automatically crops the image using an area of interest (AOI) defined by the user, which considerably enhances the overall performance. ZoneMAP also automatically re-projects and re-samples different images to a common projection plane with an equal ground sampling distance determined by the user. The open source libraries, geospatial data abstraction library (GDAL) and OpenGIS simple features reference implementation (OGR), are used to implement these procedures (GDAL 2009). 
Users' data

In addition to remote-sensed imagery that is available, ZoneMAP can also accept data that a user provides. Data collected by users, such as yield or soil electrical-conductivity (EC), can be uploaded and used in classification. The data can be either in grid text format or as a raster image. The grid format follows the common convention, i.e., the first two columns are longitude and latitude and the rest of the columns are values for different parameters. The data in text format is automatically converted to an image. A zone map that a user creates is also considered as the user's data. All the data that a user provides or creates along with their metadata are saved in a secure online database. For each creation of a set of management zones, metadata is generated automatically describing the procedure and datasets used so that the classification can be reproduced later.

The created management zones can be downloaded in one of the three formats, raster image, grid text or shape file. For each format, there are multiple projections to choose from. In addition, users can input an application rate for each zone to generate a variable rate application map.

\section{Results and discussion}

The performance of ZoneMAP was tested using data from two private farm fields, which are for crop production but not specifically designated for research. All field data collection and processing were conducted by the owners, who received no technical inputs or financial support from the project. Despite uncertainties that may be associated with this data collection policy, it is important to evaluate the performance of ZoneMAP using real data by real users. Both fields are non-irrigated. Field 1 has a clay soil and Field 2 has silty loam soil.

\section{Field 1}

The first field of an area of 96.6 ha is located in Polk County of Minnesota, USA, with soybeans planted in 2004 and wheat in 2005. A total of 93 samples were collected in the fall of 2005 after the harvest to estimate phosphorus (P), potassium (K), soil organic matter (SOM) and $\mathrm{pH}$. The distribution of sampling points is shown in Fig. 2a.

Eight Landsat images between June 2004 and August 2005 contained the field. The temporal variations of surface reflectance measured by Landsat at the wavelengths of the blue (band 1) and the NIR (band 4) along with the corresponding NDVI and GNDVI are shown in Fig. 3. Cloud cover limited the satellite coverage to the first half of the growing season for 2004 and the second half for 2005.

Due to strong absorption by chlorophyll pigments at the blue and red wavelengths, the reflectance of band 1 of Landsat typically decreases as chlorophyll concentration increases with maturation, and then increases as chlorophyll concentration decreases towards senescence. The same trend can be observed for band 3 (the red, not shown). The reflectance at the NIR, which is largely due to leaf cellular structure, typically exhibits the opposite effect. The maximum reflectance in the NIR and hence the maximum vegetation indices (NDVI and GNDVI) occurred on July 26 for 2004 and July 13 for 2005. These maximums likely occurred when crops reached full canopy. The NDVI map on July 13, 2005 is shown in Fig. 2a. 

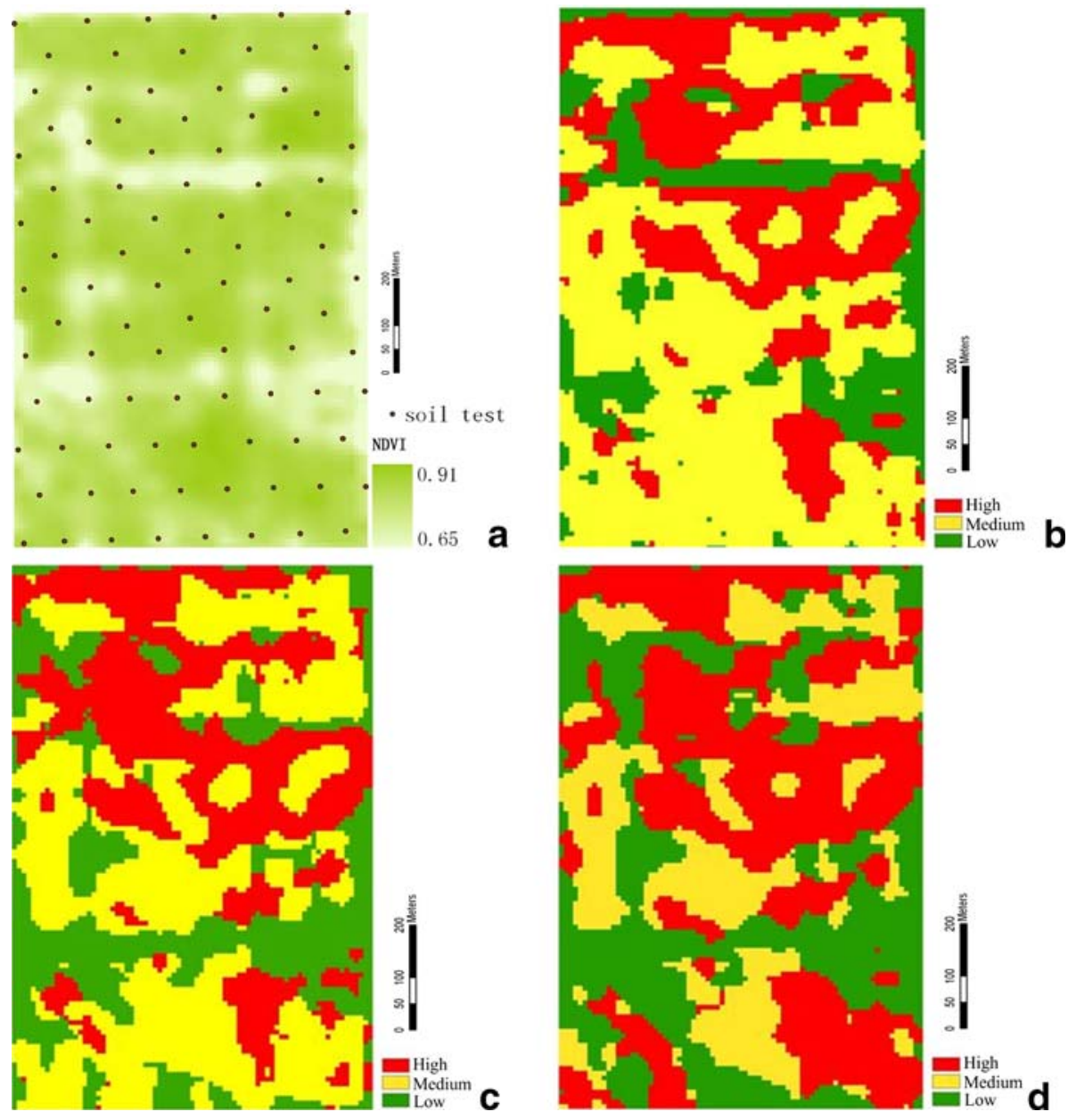

Fig. 2 a NDVI estimated from Landsat TM image on July 13, 2005 with sampling points overlaid. Two images on July 26, 2004 and July 13, 2005 were used and three zone maps were created using values from each image of NDVI (b), GNDVI (c) and band 4 (d), respectively. In the legends, high, medium and low correspond to SOM concentration

The images on July 26, 2004 and July 13, 2005, when the reflectance in the NIR and the values of NDVI and GNDVI reached their maximums for the respective year, were chosen to create three zone maps: the first used a pair of NDVI data as inputs (Fig. 2b), the second used a pair of GNDVI (Fig. 2c) and the third used a pair of the NIR values (Fig. 2d). When two NDVI images were used, the optimal number of zones determined by ZoneMAP was three, which was also used for the other two classifications. Each zone was identified by its corresponding mean SOM value, as high, medium or low. Table 1 lists the analysis of variance of soil properties ( $\mathrm{SOM}, \mathrm{pH}, \mathrm{K}$, and $\mathrm{P}$ ) as a function of management zones created using the three different datasets, respectively. The correlation coefficients were estimated with data from the image of July 13, 2005.

For management zones based on GNDVI or NIR values (Fig. 2c or d), levels of SOM were significantly different between zones 1 and 2 and zone 3 , while for management 
Fig. 3 Temporal variation of the mean (and the standard deviation) of surface reflectance by Landsat at the blue and the NIR bands and vegetation indices of NDVI and GNDVI for the growing season of 2004 (solid line) and 2005 (dashed line)

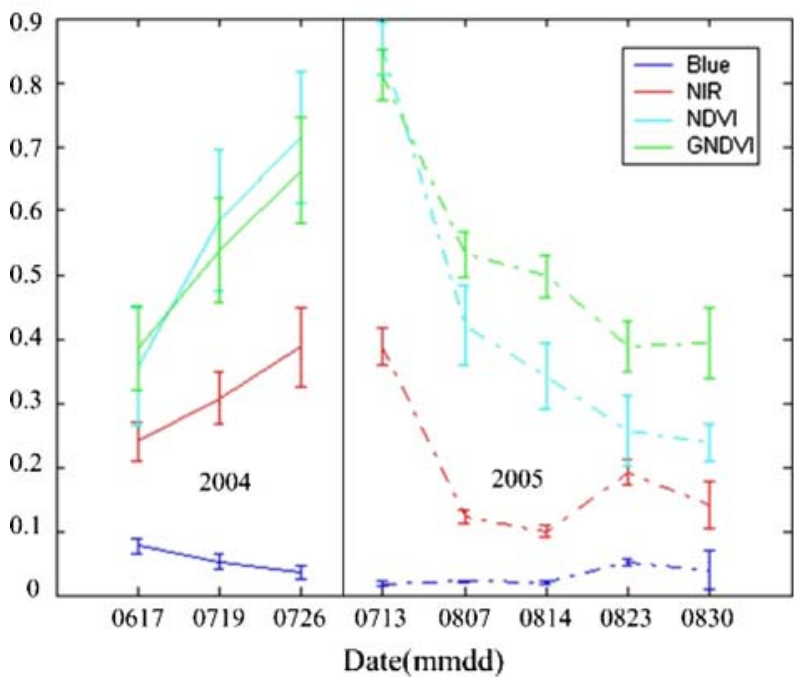

Table 1 Analysis of variance of SOM and $\mathrm{pH}$ as a function of management zones

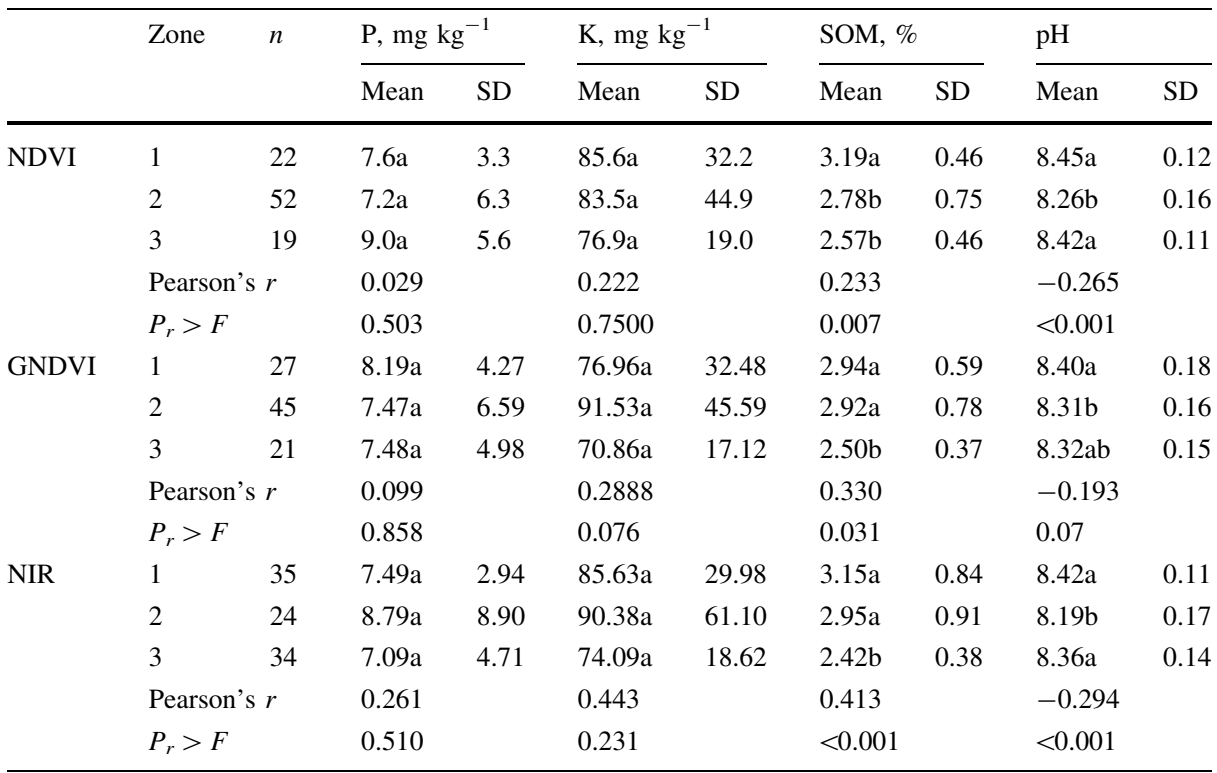

Means followed by different letters are significantly different at $p<0.05$

zones based on NDVI (Fig. 2b), levels of SOM were significantly different between zone 1 and zones 2 and 3. Relative high correlation $(\sim 0.4)$ between SOM and the reflectance at the NIR may suggest that the cellular structure of canopy correlates well with soil organic matter concentration (Wetterlind et al. 2008). This may explain that zones based on the NIR values performed better in terms of partition of SOM (Table 1). Because of the high correlation between SOM and the soil reflectance in the NIR, the spectroscopy in the NIR has been used to model the SOM (Daniel et al. 2004; Wetterlind et al. 2008). 
The $\mathrm{pH}$ values are significantly different between zone 1 and zone 2 and are similar between zone 1 and zone 3 for all three zone maps. Except for the map based on GNDVI values, the $\mathrm{pH}$ values are also significantly different between zone 3 and zone 2 . Rogovska and Blackmer (2009) found $\mathrm{pH}$ was significantly correlated with GNDVI, which was used to quantitively map soil alkalinity at a field scale so that soybean iron deficiency chlorosis can be identified and managed. The correlation between $\mathrm{pH}$ and GNDVI for Field 1 is less significant than that between $\mathrm{pH}$ and NDVI and NIR, respectively. However, the variation of $\mathrm{pH}$ in Field 1 is much limited (8.0-8.6) as compared to the variation in Rogovska and Blackmer's study, where $\mathrm{pH}$ varied from 5.4 to 8.4. In contrast to SOM, the reflectance at the NIR and the two indices co-varied with $\mathrm{pH}$ values negatively.

The differences of $\mathrm{K}$ and $\mathrm{P}$ among different zones are insignificant for all the three maps, regardless of datasets being used. It is interesting to note that the correlation between $\mathrm{K}$ and NIR reflectance is the highest among those listed in Table 1.

The results mentioned above were based on the two Landsat images acquired at the full canopy stage of the growing seasons. Classifications using images at other growing stages were also tested. Management zones created were not as effective in terms of partitioning SOM. However, they may be more effective for other applications such as fertilizer.

\section{Field 2}

The second field is located in Potter County, South Dakota, USA with an area of 45.3 ha. The rotation of crops from 2003 to 2005 was corn, sunflowers and spring wheat. Using a yield map collected in 2003 (Fig. 4a) and an NDVI map by Landsat on August 25, 2004 (Fig. 4b), the farmer created four subfield zones (Fig. 4c) to determine the application rates of urea for the year 2005. As a result of this variable-rate application, the spring wheat planted in 2005 delivered a much more uniform yield (Fig. 4d). While the mean yields of each crop were about the same, $7.33 \mathrm{t} / \mathrm{ha}$ for corn and $7.17 \mathrm{t} / \mathrm{ha}$ for spring wheat, the standard deviation (SD) was reduced from $1.93 \mathrm{t} / \mathrm{ha}$ for corn in 2003 to $1.23 \mathrm{t} / \mathrm{ha}$ for spring wheat in 2005.

Yield monitoring capability is not available to every farmer. However, spectral data from remote sensing has been shown to have potential to assess the yield variation at both large (county or sub-county) or small (within-field) scales (Doraiswamy et al. 2003, 2004; Tucker et al. 1980). It is of interest to evaluate whether replacing the yield map with satellite imagery can generate an equivalent zone map. The NDVI derived from Landsat on September 1, 2003 is shown in Fig. 4e. Visual examination of the image and the yield map of corn for 2003 (Fig. 4a) does suggest some degree of correlation. The zone map created using the NDVIs from September 12003 and August 252004 is shown in Fig. 4f. Zones 1, 3,5 and 6 roughly correspond to the zones with low, moderate, high and extra high rate in Fig. 4c, respectively.

Based on the contingency table for the two zone maps (Table 2), management zones shown in Fig. $4 \mathrm{c}$ and $\mathrm{f}$ are similar to each other with an association coefficient (estimated using Pearson's $C$ ) of 0.52 , confirming the earlier studies that spectral reflectance in the late crop growing season could be used to map crop yields (Moran et al. 1997). Differences between the two maps can also be observed, especially for zone designated as "extra high rate". This is not surprising because yield is influenced by a range of factors, some of which may not be manifested radiometrically. Tucker et al. (1980) found that a maximum of $64 \%$ of variability in a grain yield can be explained by the spectral data. This also corroborates one argument that has been frequently stressed by end users who are early 


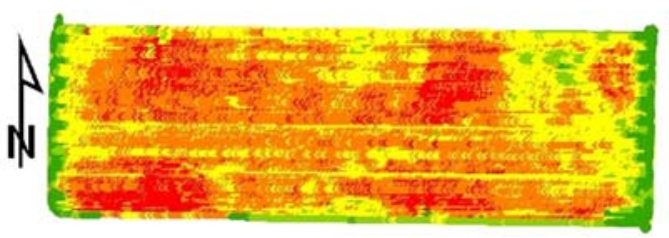

Yield $\mathrm{t} / \mathrm{ha}$

- $<2.5$

- 2.5-5.7

5. 7-7.6

- 7.6-8.9

- 8. 9-15.2

. 50 $100 \quad 200$ Meters $\mathbf{a}$
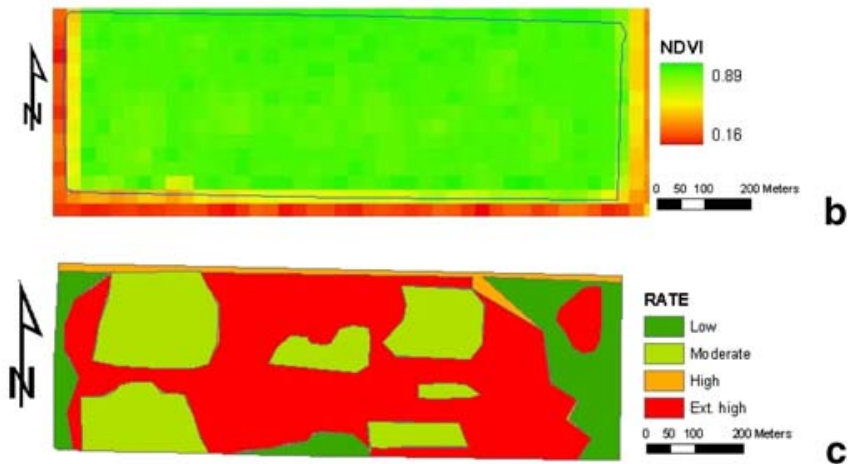

C

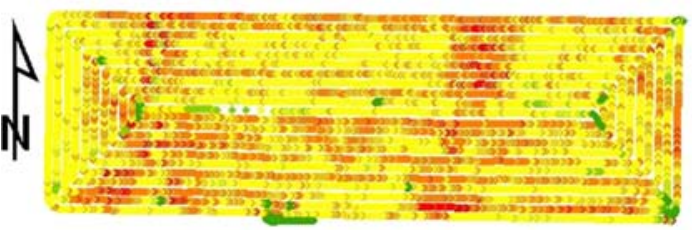

Yield tha

- $<2.5$

- 2.5-5.7

5. $7-7.6$

- 7. 6-8.9

- $8.9-15.2$

1 $50100 \quad 200$ Meters

d

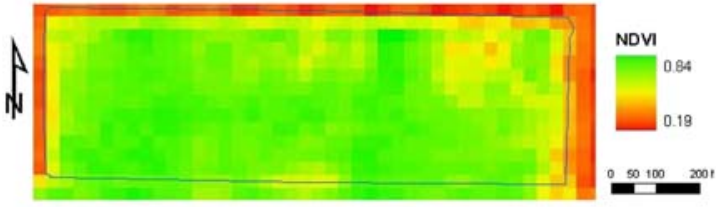

e

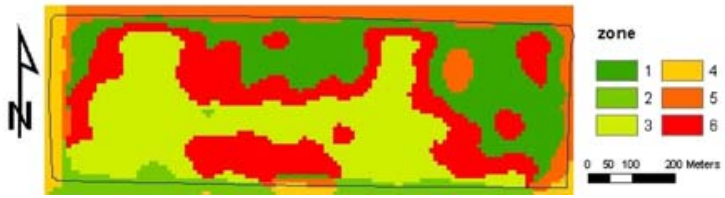

f

Fig. 4 Using 2003 yield map of corn (a) and 2004 NDVI map by Landsat of August 25, 2004 (b), the farmer created the management zones (c) as a basis for determination of variable rate fertilizer application resulting in a more uniform yield for 2005 spring wheat (d). The zone map (f) when the yield data of 2003 is replaced with NDVI data of September 1, 2003 (e)

adopters of precision agriculture, "it is critical to monitor the yield and use it in zone management".

\section{Conclusions}

ZoneMAP is a web-based decision support tool that can be used to delineate a field into zones using a variety of sources of data, which include remote sensing imagery from an 
Table 2 Contingency table for maps of management zones shown in Fig. 4c (as rows) and Fig. 4f (as columns)

\begin{tabular}{lrrrrr}
\hline Zones & Low & Moderate & High & Ext. high & Total \\
\hline 1 & 257 & 152 & 36 & 760 & 1205 \\
3 & 79 & 344 & 14 & 820 & 1257 \\
5 & 234 & 51 & 139 & 185 & 609 \\
6 & 19 & 736 & 22 & 699 & 1476 \\
Total & 589 & 1283 & 211 & 2464 & 4547 \\
\hline
\end{tabular}

$\chi^{2}=1631$, Pearson's $C=0.52$

archive that is internally linked and constantly updated and field data that users have collected. The data preparation tasks, such as format conversion, re-projection and gridding, are automatically handled by ZoneMAP. An algorithm based on the reduction of variance was developed to provide an estimate of the optimal number of zones. The effectiveness of ZoneMAP was tested with two commercial farm fields. The results showed that zones based on satellite images with highest NDVI values for the year correlated well with SOM concentration. Satellite images can also be used as a preliminary basis when a yield map is not available.

The use of ZoneMAP is limited by the coverage of data that it can access. Currently, satellite imagery that has been collected is targeted to the upper Midwest region of the US. This geographic boundary can be easily extended into other areas of the US or the world. In addition, users of ZoneMAP can upload and use their own data, which can be of any part of the world. To access ZoneMAP, go to http://zonemap.umac.org.

Acknowledgments The funding for the project was provided by NASA/USRA through grants NCC8-259 and 03450-08. We thank Mr. Dan Forgey, Mr. Gary Wagner and many other end users for sharing their data as well as knowledge. Without their help this research would not be possible. Funding for Lijian Shi was provided through NASA grant NNX06AE16G. Comments from two anonymous reviewers are greatly appreciated.

Open Access This article is distributed under the terms of the Creative Commons Attribution Noncommercial License which permits any noncommercial use, distribution, and reproduction in any medium, provided the original author(s) and source are credited.

\section{References}

Bégué, A., Todoroff, P., \& Pater, J. (2008). Multi-time scale analysis of sugarcane within-field variability: Improved crop diagnosis using satellite time series. Precision Agriculture, 9, 161-171.

Bhatti, A. U., Mulla, D. J., \& Frazier, B. E. (1991). Estimation of soil properties and wheat yields on complex eroded hills using geostatistics and thematic mapper images. Remote Sensing of Environment, $37,181-191$.

Brock, A., Brouder, S. M., Blumhoff, G., \& Hofmann, B. S. (2005). Defining yield-based management zones for corn-soybean rotations. Agronomy Journal, 97, 1115-1128. doi:10.2134/agronj2004.0220.

Burrough, P. A. (1989). Fuzzy mathematical methods for soil survey and land evaluation. European Journal of Soil Science, 40, 477-492.

Burrough, P. A., MacMillan, R. A., \& Deursen, W. V. (1992). Fuzzy classification methods for determining land suitability from soil profile observations and topography. European Journal of Soil Science, 43, 193-210.

Daniel, K. W., Tripathi, N. K., Honda, K., \& Apisit, E. (2004). Analysis of VNIR (400-1100 nm) spectral signatures for estimation of soil organic matter in tropical soils of Thailand. International Journal of Remote Sensing, 25, 643-652. 
Doraiswamy, P. C., Hatfield, J. L., Jackson, T. J., Akhmedov, B., Prueger, J., \& Stern, A. (2004). Crop condition and yield simulations using Landsat and MODIS. Remote Sensing of Environment, 92, $548-559$.

Doraiswamy, P. C., Moulin, S., Cook, P. W., \& Stern, A. (2003). Crop yield assessment from remote sensing. Photogrammetric Engineering \& Remote Sensing, 69, 665-674.

Fleming, K. L., Westfall, D. G., Wiens, D. W., \& Brodahl, M. C. (2000). Evaluating farmer defined management zone maps for variable rate fertilizer application. Precision Agriculture, 2, 201-215.

Franzen, D. W., Hopkins, D. H., Sweeney, M. D., Ulmer, M. K., \& Halvorson, A. D. (2002). Evaluation of soil survey scale for zone development of site-specific nitrogen management. Agronomy Journal, 94, 381-389.

Fridgen, J. J., Kitchen, N. R., Sudduth, K. A., Drummond, S. T., Wiebold, W. J., \& Fraisse, C. W. (2004). Management zone analyst (MZA): Software for subfield management zone delineation. Agronomy Journal, 96, 100-108.

GDAL. (2009). http://www.gdal.org/. Accessed July 7, 2009.

Gitelson, A. A., Kaufman, Y. J., \& Merzlyak, M. N. (1996). Use of a green channel in remote sensing of global vegetation from EOS-MODIS. Remote Sensing of Environment, 58, 289-298.

Johnson, C. K., Mortensen, D. A., Wienhold, B. J., Shanahan, J. F., \& Doran, J. W. (2003). Site-specific management zones based on soil electrical conductivity in a semiarid cropping system. Agronomy Journal, 95, 303-315.

Lark, R. M., \& Stafford, J. V. (1997). Classification as a first step in the interpretation of temporal and spatial variation of crop yield. Annals of Applied Biology, 130, 111-121.

Metternicht, G. (2003). Vegetation indices derived from high-resolution airborne videography for precision crop management. International Journal of Remote Sensing, 24, 2855-2877.

Moran, M. S., Inoue, Y., \& Barnes, E. M. (1997). Opportunities and limitations for image-based remote sensing in precision crop management. Remote Sensing of Environment, 61, 319-346.

Moreenthaler, G. W., Khatib, N., \& Kim, B. (2003). Incorporating a constrained optimization algorithm into remote sensing/precision agriculture methodology. Acta Astronautica, 53, 429-437.

Odeh, I. O. A., Chittleborough, D. J., \& McBratney, A. B. (1992). Soil pattern recognition with fuzzyc-means: Application to classification and soil-landform interrelationships. Soil Science Society of America Journal, 56, 505-516.

Read, J. J., Tarpley, L., McKinion, J. M., \& Reddy, K. R. (2002). Narrow-waveband reflectance ratios for remote estimation of nitrogen status in cotton. Journal of Environmental Quality, 31, 1442-1452.

Rogovska, N., \& Blackmer, A. (2009). Remote sensing of soybean canopy as a tool to map high $\mathrm{pH}$, calcareous soils at field scale. Precision Agriculture, 10, 175-187.

Salisbury, J. W., \& D’Aria, D. M. (1992). Infrared $(8-14 \mu \mathrm{m})$ remote sensing of soil particle size. Remote Sensing of Environment, 42, 157-165.

Seelan, S. K., Laguette, S., Casady, G. M., \& Seielstad, G. A. (2003). Remote sensing applications for precision agriculture: A learning community approach. Remote Sensing of Environment, 88, 157-169.

Sullivan, D. G., Shaw, J. N., \& Rickman, D. (2005). IKONOS imagery to estimate surface soil property variability in two Alabama physiographies. Soil Science Society of America Journal, 69, 1789-1798. doi:10.2136/sssaj2005.0071.

Tucker, C. J., Holben, B. N., Elgin, J. H., Jr., \& McMurtrey, J. E. (1980). Relationship of spectral data to grain yield variation. Photogrammetric Engineering \& Remote Sensing, 46, 657-666.

Wetterlind, J., Stenberg, B., \& Söderström, M. (2008). The use of near infrared (NIR) spectroscopy to improve soil mapping at the farm scale. Precision Agriculture, 9, 57-69.

Zhang, N., Wang, M., \& Wang, N. (2002). Precision agriculture-a worldwide overview. Computers and Electronics in Agriculture, 36, 113-132. 